\title{
Aktuelles Programm der DGHO Frühjahrstagung 2009, Berlin
}

\section{Donnerstag, 12.03.2009}

\section{2:30-14:30 Beiratssitzung}

14:30-15:00 Pause

15:00-16:30 Fortbildungsveranstaltung der GMIHO mbH

16:30-17:00 Pause

17:00-19:00 Biomarker

Vorsitz: U. Keilholz, Berlin und U. Dührsen, Essen

- Repair-Mechanismen, A. Hoffmann, Essen

- K-RAS-Mutationen und andere Biomarker bei Tumoren, A. Tannapfel, Bochum

- Biomarkergetriebene Entwicklung: Chance/Irrweg? A.-R. Hanauske, USA (a. A.)

- Expression der Topoisomerase alpha als prädiktiver Marker, M. Schuler, Essen

19:00-19:30 Pause

19:30-21:30 Politischer Auftakt

«Gesundheitspolitik in Zeiten finanzieller

Anspannung» Zugang zu Innovationen für alle? Moderation: Sabine Rieser, Deutsches Ärzteblatt

ab 21:30 Empfang

Freitag, 13.03.2009

8:30-10:30 AK Palliativmedizin

8:30-10:30 AK Nicht -Maligne Hämatologie
8:30-10:30 Wie sicher ist die Arzneimittelsicherheit?

Vorsitz: G. Ehninger, Dresden

- Arzneimittelentwicklung und Studien, D. Hoelzer, Frankfurt a. M.

- Politische Überregulation: Gefährdung und nicht Gewinn für den Patienten! V. Diehl, Köln

- Arzneimitteltherapiesicherheit in der Onkologie: W.-D. Ludwig, Berlin

- Entwicklung der Zahlen der IITs in Deutschland, NN

10:30-11:00 Pause

11:00-12:30 Neues aus der Hämatologie

Satelliten-Symposium der Firma Celgene GmbH

- Myelodysplasie und AML, U. Platzbecker, Dresden

- B-Zell-Neoplasien: Lymphome, Myelom und CLL, U. Dührsen, Essen

12:30-13:00 Pause

13:00-14:30 Onkologische Innovationen im Spannungsfeld von Wissen: Gewissen und wirtschaftlichen Rahmenbedingungen, Symposium der Roche Pharma AG,

Vorsitz: T. Hegemann

- Medizinisch machbar und indiziert: ethische Überlegungen zur evidenzbasierten Medizin, D. Strech, Hannover

- Medizinisch machbar, aber zu teuer? Gesundheitskosten im europäischen Vergleich, M. Schlander, Mannheim

\section{KARGER}

Fax +497614520714

Information@Karger.de

www.karger.com (c) 2009 S. Karger GmbH, Freiburg

Accessible online at:

www.karger.com/onk 
- Medizinisch machbar und effizient: Kostenmanagement durch Qualitätsmanagement, M. Schrappe, Frankfurt a.M.

Podiumsgespräch und Diskussion

- Machbar und erprobt: Erfahrungen mit dem integrierten Versorgungsvertrag Maligne Lymphome, M. Hallek, Köln und J. Münstermann, Siegburg

14:30-15:00 Pause

Freitag, 13.03.2009

15:00-16:30 Qualitätssicherung - Struktur/Prozess/Ergebnisqualität

Vorsitz: A. Hochhaus, Mannheim und M. Schuler, Essen

- Überblick, M. Freund, Rostock

- Welche Qualität haben Leitlinien und ihre Entwicklung? D. Arnold, Halle/Saale

- Oncopedia, G. Folprecht, Dresden

16:30-17:00 Pause

17:00-19:00 Stahl, Strahl oder Chemie

Karzinome des Ösophagus und Magen

Vorsitz: S. Hegewisch-Becker, Hamburg und U. Keilholz, Berlin

- Fortgeschrittenes Ösophagus-Ca, J. Dunst, Lübeck

- Innovative Ansätze und Studien beim lokal fortgeschrittenen

- Ösophagus- und Magenkarzinom, F. Lordick, Heidelberg

- Multimodale Therapie des Ösophaguskarzinoms, M. Stahl, Essen

- Ist die alleinige operative Therapie bei Ösophagus- und Magenkarzinomen noch gerechtfertigt?

K. Ridwelski, Magdeburg

ab 20:00 Get Together Party in den neuen Büroräumen der DGHO

\section{Samstag, 14.03.2009}

8:30-10:30 Onkologische Zentren - Workshop Vorsitz: M. Freund, Rostock und M. Wilhlem, Nürnberg

- Erfahrungsaustausch

- Datentransfer/Schnittstellenproblematik

- HOME-CARE - DGHO (Onlinepräsentation) Ein Internet-basiertes Dokumentationssystem zur Qualitätssicherung ambulanter Palliativversorgung, W. Freier, Hildesheim

08:30-10:30 AK Labor

08:30-10:30 AK DRG: Dokumentation und Kodierung

08:30-10:30 AK Onkologische Rehabilitation

10:30-11:00 Pause

11:00-13:00 Aktuelle Therapieempfehlungen

Vorsitz: W. Berdel, Münster und S. Schmitz, Köln

- Sequenztherapie des metastasierten Ovarialkarzinoms, D. Chatsiproios, Mannheim

- CML, A. Hochhaus, Mannheim

- Algorithmus beim Multiplen Myelom, H. Einsele, Würzburg

- Optimierungspotentiale bei der Behandlung der chronisch myeloischen Leukämie, J. Hasford, München

- Präsentation einer GfK-Studie, P. Kuester, Nürnberg

11:00-13:00 AK Arzneimittelsicherheit

11:30-13:00 Arbeitskreis Klinische Studien (AKS)

13:00-13:30 Pause

ab 13:30 Ordinarientreffen (nur auf Einladung)

Stand: $\quad$ 27.01.2009/Änderungen vorbehalten. 